\title{
A relação entre eventos climáticos extremos e desastres ambientais fluviais no
}

\author{
Amazonas \\ The relation between extreme climate events and river environmental disasters in Amazonas \\ La relación entre los fenómenos meteorológicos extremos y los desastres ambientales fluviales en el
}

Amazonas

Recebido: 05/07/2021 | Revisado: 14/07/2021 | Aceito: 16/07/2021 | Publicado: 25/07/2021

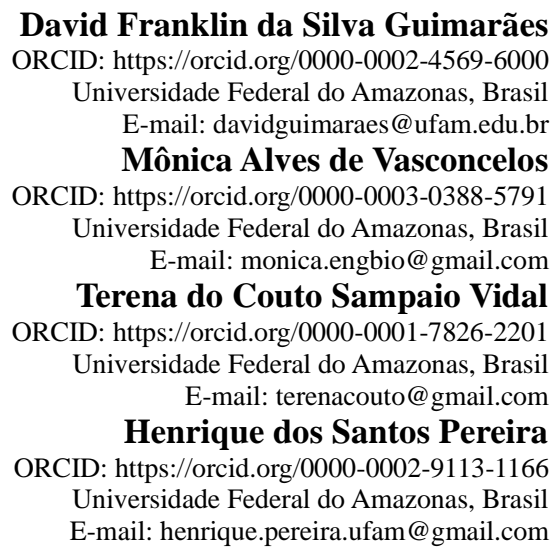

\section{Resumo}

O objetivo deste estudo foi investigar os padrões de distribuição geográfica e temporal dos desastres ambientais fluviais a partir do comparativo das cotas fluviométricas com os limiares dos eventos climáticos extremos fluviais registrados no Amazonas. A metodologia consistiu em uma abordagem quali-quantitativa com o processamento estatístico de dados históricos de cotas fluviais das sub-regiões do estado do Amazonas, obtidos junto à Agência Nacional de Águas, processamento dos dados sobre desastres ambientais obtidos junto ao Sistema Nacional de Proteção e Defesa Civil e revisão bibliográfica sobre o assunto. De acordo com os registros das estações fluviométricas estudadas, nos últimos anos, houve uma maior frequência de eventos extremos fluviais no Amazonas. Os anos com maior ocorrência de eventos extremos de seca foram 1998, 2005 e 2010 e para os eventos extremos de inundação foram 2009, 2012, 2013, 2014 e 2015. O período analisado para desastres ambientais (2005-2016) fluviais revelou a mesma ocorrência dos eventos extremos climáticos. Logo, há uma forte ligação entre a ocorrência de eventos extremos fluviais com a os desastres ambientais. Entretanto, em alguns locais os eventos não precisam ultrapassar os limiares de extremos para gerar impactos sociais e econômicos às populações locais e serem reconhecidos como desastres. Todas as sub-regiões do Amazonas já sofrem as consequências provenientes da alteração do padrão sazonal dos rios, gerando uma maior ocorrência de eventos extremos hidrológicos e, consequentemente, de desastres ambientais fluviais.

Palavras-chave: Sazonalidade; Mudança climática; Amazônia; Inundação; Seca; Desastres naturais.

\begin{abstract}
The aim of this research was to investigate the patterns of the geographical and temporal distribution of environmental disasters since the extreme fluvial climatic events recorded in the Amazonas. The methodology consisted of a qualitative and quantitative approach and the statistical processing of historical data of river quotas in the subregions of the state of Amazonas. Data processing of environmental disasters was obtained in the National System of Protection and Civil Defense, and in a literature review on the subject. In recent years, there has been a higher frequency of extreme river events in the Amazonas. In the studied seasons, the years with the highest occurrence of extreme events were 1998, 2005, and 2010 for drought and 2009, 2012, 2013, 2014, and 2015 for a flood. The period analyzed for environmental disasters (2005-2016) revealed the same occurrence of extreme weather events. Thereby, there is a strong link between the occurrence of extreme river events and environmental disasters, however, in some places, the climatic events do not need to exceed normal limits to generate social and economic impacts for local populations or been recognized as disasters. All subregions of the Amazonas are already affected by the consequences of seasonal changes in the rivers. These changes are caused by global warming, generating a greater occurrence of extreme hydrological events and, consequently, river environmental disasters.
\end{abstract}

Keywords: Seasonality; Global warming; Amazon; Drought; Flood; Natural disasters. 


\begin{abstract}
Resumen
El objetivo de este estudio fue investigar los patrones de distribución geográfica y temporal de los desastres ambientales fluviales a partir de la comparación de las cuotas fluviométricas con los umbrales de eventos climáticos fluviales extremos registrados en el Amazonas. La metodología consistió en un enfoque cuali-cuantitativo contando con un procesamiento estadístico de los datos históricos de las cuotas fluviales de las subregiones del estado de Amazonas los cuales fueron obtenidos de la Agencia Nacional del Agua. El procesamiento de los datos sobre desastres ambientales fue obtenido del Sistema Nacional de Protección y Defensa Civil y la revisión de la literatura sobre el tema. De acuerdo con los registros de las estaciones fluviométricas estudiadas, en los últimos años ha habido una mayor frecuencia de eventos fluviales extremos en el Amazonas. Los años con mayor ocurrencia de eventos de sequía extrema fueron 1998, 2005 y 2010 y para los eventos de inundación extrema fueron 2009, 2012, 2013, 2014 y 2015. El período analizado para las catástrofes ambientales fluviales (2005-2016) reveló la misma ocurrencia de eventos climáticos extremos. Por lo tanto, existe un fuerte vínculo entre la ocurrencia de eventos fluviales extremos y los desastres ambientales. Sin embargo, en algunos lugares no es necesario que los eventos superen los umbrales de los extremos para generar impactos sociales y económicos en las poblaciones locales y ser reconocidos como desastres. Todas las subregiones de la Amazonia ya sufren las consecuencias de la alteración del patrón estacional de los ríos, generando una mayor ocurrencia de eventos hidrológicos extremos y, en consecuencia, de desastres ambientales fluviales.
\end{abstract}

Palabras clave: Estacionalidad; Cambio climático; Amazonia; Inundación; Sequía; Desastres naturales.

\title{
1. Introdução
}

Os eventos extremos climáticos não ocorrem de forma isolada, eles fazem parte de um padrão maior, tendo conexões com outros lugares e em escala global (Da Silva Dias, 2014). De acordo com o IPCC (2007), a mudança climática não pode ser relacionada aos eventos extremos que ocorrem de forma isolada, visto que os extremos ocorrem naturalmente, mas, a persistência de um padrão de tempo da ocorrência desses eventos pode ser atribuída às alterações do clima.

O padrão sazonal dos rios amazônicos vem sendo alterado devido à mudança climática global (Marengo et al., 2008), resultando em uma maior frequência de ocorrências de eventos extremos fluviais e impactando diretamente a vida das populações ribeirinhas. Essa nova conjuntura gerou insegurança ambiental expondo tais comunidades a uma maior situação de risco. Os eventos extremos fluviais representam a superação do padrão de normalidade, tanta para cotas mínimas como para máximas, da sazonalidade dos rios. Na Amazônia, os eventos extremos fluviais vinham sendo atribuídos aos eventos climáticos El Niño e La Niña que correspondem ao aquecimento e resfriamento das águas do Oceano Pacífico, respectivamente, gerando aumento ou redução da precipitação, bem como alterações na descarga e níveis dos rios (Zeng, 2008).

No Amazonas, os eventos extremos fluviais passaram a ter maior relevância e preocupação do poder público a partir da ocorrência da seca de 2005, que gerou inúmeras implicações socioambientais no estado, sendo a mesma difundida pelos meios de comunicação como desastre ambiental (Rodrigues, 2013). Nas várzeas, a água é o agente transformador e regulador da vida do homem amazônico, possuindo uma centralidade na vida das populações, principalmente em relação à sazonalidade de seus rios (Tocantins, 2000; Sternberg, 1997). Essas oscilações sazonais nos regimes dos rios amazônicos, com o decorrer do tempo, obrigaram a população ribeirinha a construir um modo de vida adaptado aos eventos hidrológicos, porém, atualmente, essas populações vêm sofrendo intensamente com os eventos extremos fluviais (Filizola et al., 2006).

No Brasil, os desastres são classificados pela Secretaria Nacional de Proteção e Defesa Civil a partir da Codificação Brasileira de Desastres (COBRADE). Na COBRADE os desastres oriundos de perigos naturais, como os eventos extremos fluviais, são conhecidos apenas como desastres naturais (natural hazards) (UNISDR, 2009). O termo hazard traduzido como perigo pelos meios acadêmicos é compreendido pelos meios técnicos, especialmente a defesa civil, como ameaça (Saito et al., 2015). A tradução como ameaça deturpa o sentido de hazard, visto que ameaça está ligada a intenção humana de punir ou prejudicar (Saito et al., 2015).

Para Carvalho (2013) os desastres devem ser identificados para além da vontade divina ou eminentemente natural, demonstrando os aspectos sociais e humanos dos desastres. Logo, deve-se considerar a complexidade que envolve a ocorrência 
desses desastres que permeiam o fenômeno natural (perigo), mas, sobretudo, a vulnerabilidade social das populações propensas aos seus impactos.

Os desastres ambientais fluviais podem ser classificados quanto à forma de ocorrência em bruscos (súbitos) ou graduais. As inundações bruscas, por exemplo, são resultados de chuvas intensas e/ou prolongadas provocando impactos às populações vulneráveis de forma imediata, já as inundações graduais, como as ocorridas na Amazônia, são resultados de diversos fatores climáticos que intensificam o período da estação cheia provocando inúmeros problemas para as populações ribeirinhas (Freitas et al., 2014).

Para contribuir com essa discussão, este estudo teve como objetivo investigar os padrões de distribuição geográfica e temporal dos eventos climáticos extremos fluviais, a ocorrência do "perigo", e as correlações com os desastres ambientais fluviais registrados no Amazonas.

\section{Metodologia}

A presente pesquisa contou com um acervo de dados secundários obtidos de revisão bibliográfica, estudo documental e dados de agências e sistemas oficiais. A revisão bibliográfica nessa pesquisa abrange a busca de informações em periódicos, livros e outras formas de comunicação científica que abordam sobre a sazonalidade dos rios no estado do Amazonas, bem como sobre os eventos extremos fluviais e desastres ambientais.

Para o estabelecimento dos limiares extremos das sub-regiões do estado do Amazonas foram coletadas informaç̃es das cotas fluviométricas dos períodos de inundação e vazante de uma estação de cada sub-região, na base de dados do HidroWeb da Agência Nacional de Águas (ANA) (Figura 1).

Figura 1. Mapa com as estações selecionadas.

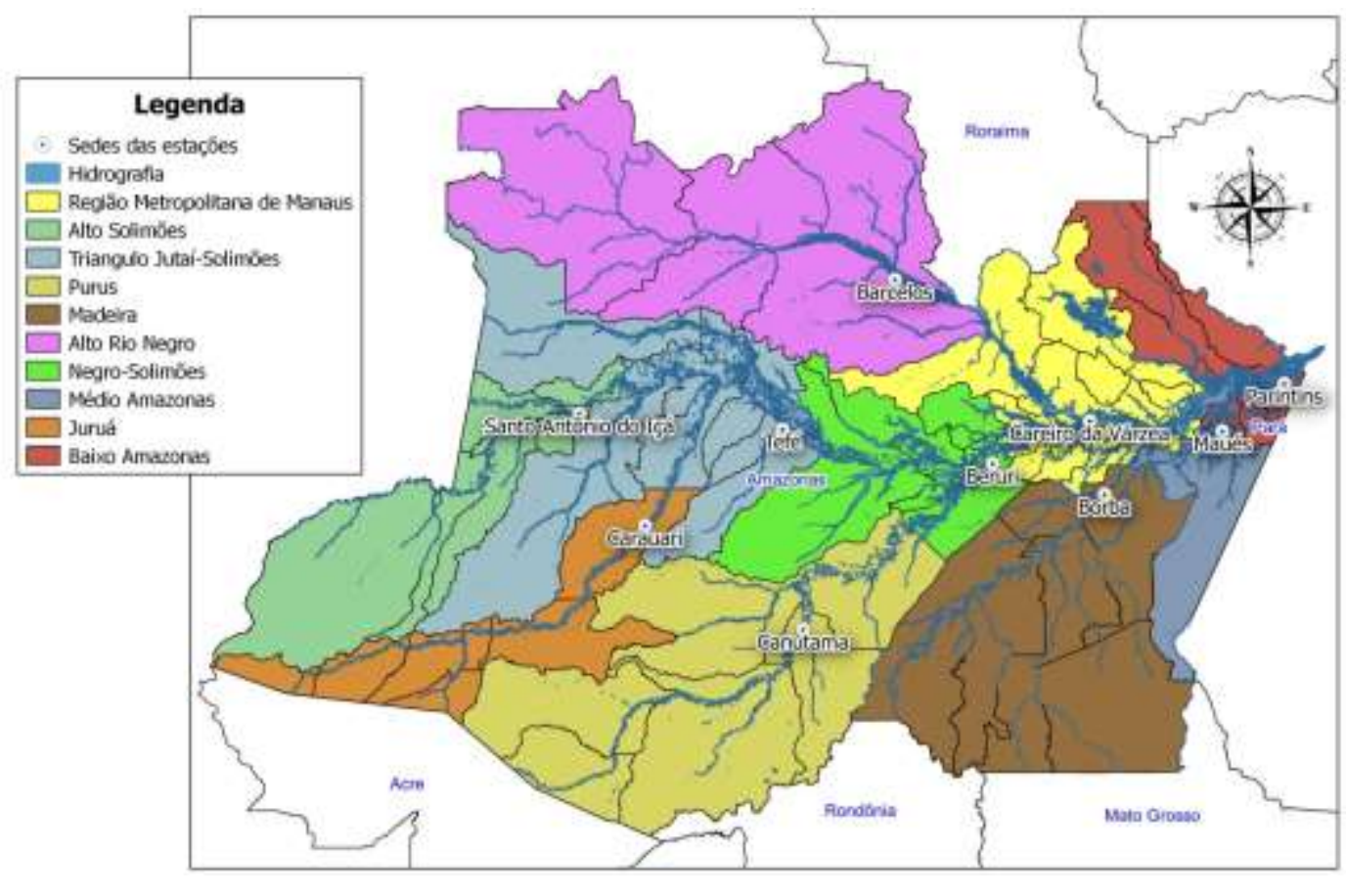

Fonte: Guimarães (2021).

Para esse estudo foram utilizados dados de estações fluviométricas dos municípios de Barcelos (Alto Rio Negro), Beruri (Médio Amazonas), Borba (Madeira), Canutama (Purus), Carauari (Juruá), Careiro da Várzea (Região metropolitana de 
Manaus), Maués (Médio Amazonas), Parintins (Baixo Amazonas), Santo Antônio do Içá (Alto Solimões) e Tefé (Triângulo Jutaí-Solimões). O processamento foi realizado com os dados brutos mensais de cada estação, esses dados foram analisados previamente para verificação de possíveis erros humanos na tabulação, bem como exclusão de dados com informações incompletas. Cabe ressaltar que há ausência de dados fluviométricos em alguns meses para a maioria das estações.

O critério para a seleção das estações foi a disponibilidade da série histórica de no mínimo 25 anos, para o estabelecimento de limiares entre os eventos fluviais normais e os críticos, bem como a localização das mesmas mais a jusante. A escolha de estações mais a jusante deve-se a melhor representação que esta pode dar sobre a realidade da sub-região, já que a estação recebe contribuição dos rios que se localizam mais a montante.

Os limiares para determinar os eventos extremos fluviais foram calculados a partir da seguinte equação (Naghettini \& Pinto, 2007):

$$
e q 1=\frac{\left(\sum r\right)}{N r} \pm \sigma_{f}
$$

Onde:

$\sum r$ é a soma dos registros de toda a série histórica da sub-região;

Nr é o número de registros;

$\sigma_{f}$ é o desvio padrão.

A adição foi empregada para identificação de limiares dos eventos de inundação e a subtração aos de vazante. Os dados obtidos foram processados em planilhas eletrônicas para obtenção de estatística descritiva e o estabelecimento dos limiares extremos máximos e mínimos.

Para analisar a ocorrência distribuição de desastres foram utilizados dados disponíveis no Sistema Integrado de Informações sobre Desastres (S2iD) para os 62 municípios do estado do Amazonas. Esses dados foram tabulados em uma planilha para a quantificação da ocorrência de desastres ambientais nos municípios do estado. A escala temporal utilizada para essa etapa da pesquisa foi o período de 2005 a 2017, devido a disponibilidade dos dados. Os dados foram acessados no período de janeiro a dezembro de 2017 na plataforma do Sistema Integrado de Informações sobre Desastres.

Foram tabulados todos os dados de desastres relacionados de forma direta ou indiretamente à dinâmica hidrológica. E analisados os dados de enxurradas, deslizamentos, corridas de massa, erosão fluvial, estiagem, tempestade local/convectiva, colapso em edificações, inundação, alagamento e enchentes. A partir da sistematização das informações foram estabelecidas as frequências absolutas para cada desastre.

\section{Resultados e Discussão}

\subsection{Identificação dos eventos extremos nas sub-regiões do Amazonas}

Ao processar os dados das estações fluviométricas das áreas de estudo foi possível identificar os limiares dos eventos extremos fluviais nas áreas estudadas (Tabela 1). 
Tabela 1. Limiares de mínimos e máximos para as sub-regiões estudadas.

\begin{tabular}{|c|c|c|c|c|c|c|}
\hline \multirow[b]{2}{*}{$\mathbf{N}^{\mathbf{0}}$} & \multirow[b]{2}{*}{$\begin{array}{l}\text { Código da } \\
\text { Estação }\end{array}$} & \multirow[b]{2}{*}{ Município } & \multirow[b]{2}{*}{ Sub-região } & \multirow{2}{*}{$\begin{array}{c}\text { Período } \\
\text { Registrado } \\
\text { (anos) }\end{array}$} & \multicolumn{2}{|c|}{ Limiares $(\mathrm{cm})^{*}$} \\
\hline & & & & & $\begin{array}{l}\text { Máximos } \\
\text { (cm) }\end{array}$ & $\begin{array}{c}\text { Mínimos } \\
\text { (cm) }\end{array}$ \\
\hline 1 & 15900000 & Borba & Madeira & 50 & 2156,16 & 818,07 \\
\hline 2 & 12900001 & Tefé & Triângulo Jutaí-Solimões-Juruá & 34 & 1493,39 & 122,20 \\
\hline 3 & 16350002 & Parintins & Baixo Amazonas & 45 & 958,01 & $-49,57$ \\
\hline 4 & 14480002 & Barcelos & Alto Rio Negro & 49 & 965,63 & 143,75 \\
\hline 5 & 15040000 & Careiro da Várzea & $\begin{array}{c}\text { Região Metropolitana de } \\
\text { Manaus }\end{array}$ & 39 & 1699,28 & 324,29 \\
\hline 6 & 13990000 & Beruri & Rio Negro-Solimões & 48 & 2152,40 & 780,13 \\
\hline 7 & 12840000 & Carauari & Juruá & 46 & 1486,59 & 0,62 \\
\hline 8 & 16250000 & Maués & Médio Amazonas & 27 & 1205,28 & 61,14 \\
\hline 9 & 11500000 & Santo Antônio do Içá & Alto Solimões & 44 & 1397,58 & 154,00 \\
\hline 10 & 38800000 & Canutama & Purus & 45 & 2452,24 & 774,29 \\
\hline
\end{tabular}

*As cotas indicadas na tabela acima são valores associados a uma referência de nível local e arbitrária, válida para as réguas linimétricas das estações. Fonte: Autores.

A partir do estabelecimento dos limiares de normalidade das inundações e vazantes nas diferentes sub-regiões foi possível a identificação dos eventos extremos fluviais nas estações estudadas (Figura 2).

No período analisado, o município de Borba (Figura 2A), Sub-região do Madeira, obteve um total de onze eventos extremos fluviais, sendo seis de inundação e cinco de vazante. No rio Madeira, as condições hidrológicas são controladas pelas características geomorfológicas da região, dessa forma o rio em municípios como o de Humaitá tem pouca variação em comparação com outros municípios banhados por esse rio (Muniz \& Filizola, 2014). Em Borba também se percebe uma baixa variação das cotas fluviais. Entretanto, é possível detectar a alta frequência de eventos extremos fluviais com destaque para os anos de 2008 e 2014, onde foram registradas a maior seca e maior inundação, respectivamente.

Já o município de Tefé (Figura 2B), Sub-região do Triângulo Jutaí-Solimões-Juruá, obteve no período analisado dez eventos extremos fluviais, sendo a distribuição desses iguais para inundação e seca. O município de Barcelos, Sub-região do Alto Rio Negro apresentou o maior número de ocorrência de eventos extremos desse estudo, quinze, sete de inundação e oito de vazante. No município de Tefé, na sub-região Triângulo Jutaí-Solimões-Juruá, os estudos de Jardim-Lima et al. (2005), sugerem que as características geomorfológicas dos cursos da água da região têm uma forte relação com a área de inundação da região próximo a Reserva de Desenvolvimento Sustentável Mamirauá. Nessa sub-região o maior evento de inundação ocorreu no ano de 2015 e o de seca em 2010. Das estações estudadas as cotas máximas e mínimas de Tefé possuíram o maior desvio padrão, sendo $108,4 \mathrm{~cm}$ para máximas e $229,5 \mathrm{~cm}$ para mínimas. 
Figura 2. Cotagramas fluviométricos anuais das estações estudadas.

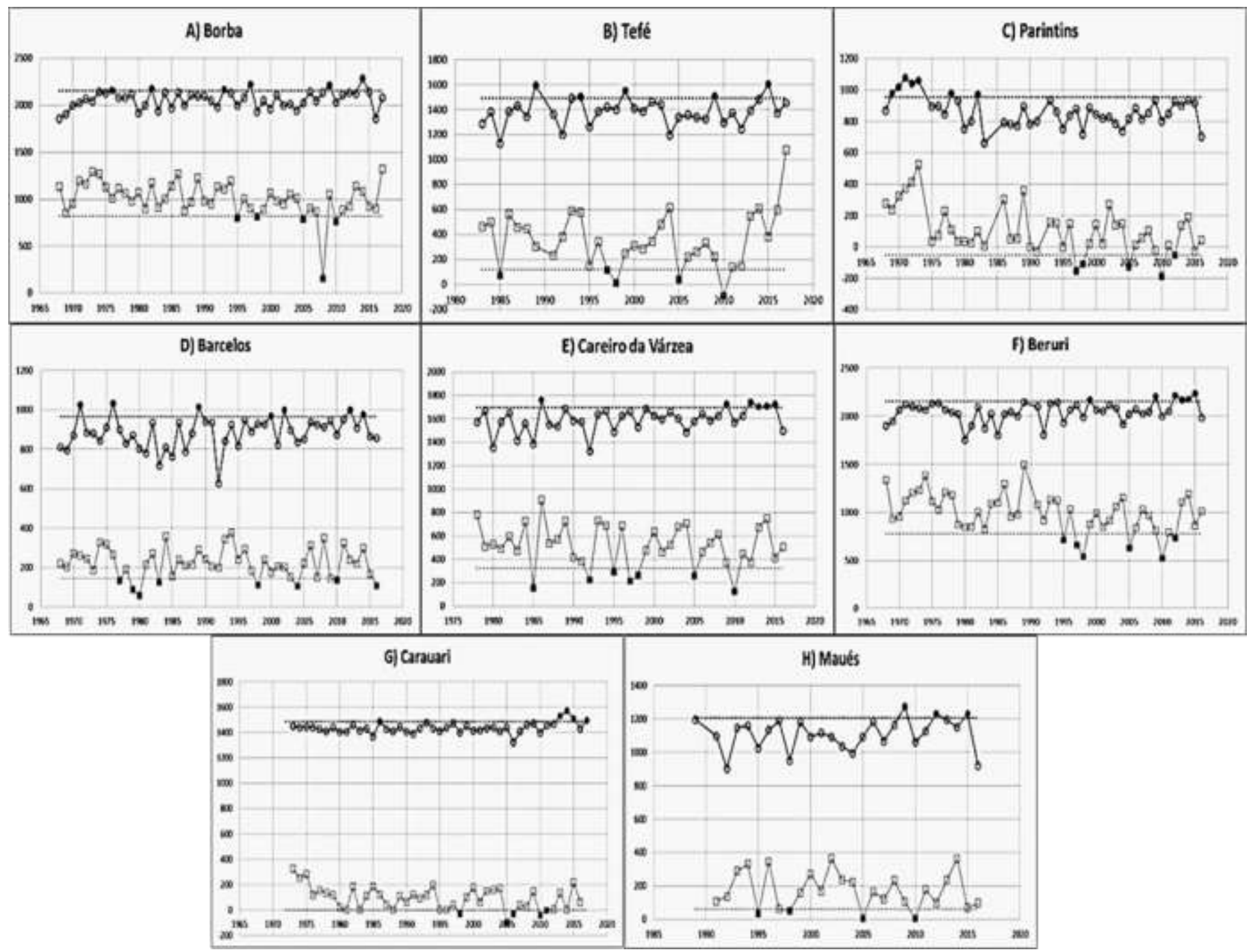

Fonte: Autores (2021).

O município de Parintins (Figura 2C), Sub-região do Baixo Amazonas, possui a ocorrência de eventos extremos de inundação concentrada no período 1969 a 1978, totalizando sete eventos nesse período, já as vazantes somam cinco ocorrências. No município de Parintins, sub-região do Baixo Amazonas, é possível comprovar esses relatos, onde há a concentração dos eventos extremos da estação selecionada nesse período. Cabe ressaltar que mesmo não ultrapassando os limiares máximos, o município de Parintins teve cinco cotas nos últimos dez anos que estiveram próximos de ultrapassar o limite de normalidade para essa estação.

Na Sub-região do Médio Amazonas, o município de Maués, com o menor número de registros, teve sete eventos extremos fluviais, três de inundação e quatro de vazante. Na região do Médio Amazonas e Baixo Solimões existem relatos da ocorrência de muitas inundações no período de 1950 a 1980 (Nascimento, 2016). Na sub-região do Médio Amazonas a falta de uma série histórica mais completa impossibilitou a análise dos anos do período de 1950 a 1980 . Mesmo assim, no município de Maués foi registrada uma maior frequência de eventos extremos fluviais a partir do ano 2000, principalmente de inundação.

O município de Careiro da Várzea, Sub-região Metropolitana de Manaus, no período analisado teve a ocorrência de doze eventos extremos, sendo proporcionalmente distribuído para inundações e secas (Figura 2E). Assim como no Careiro da Várzea, o município de Beruri, Sub-região Rio Negro-Solimões, apresenta anos semelhantes de inundação (2009, 2012, 2013, 
2014 e 2015). Nessa estação foram totalizados onze eventos extremos fluviais, sendo seis inundações e cinco vazantes. Nas sub-regiões Metropolitana de Manaus e Rio Negro-Solimões, a contribuição dos rios Solimões, Madeira e Negro geram um aumento na vazão e na superfície de drenagem do rio Amazonas, levando essas a possuírem características semelhantes. Nessas regiões foi observada uma maior frequência de inundações no período de 2009 a 2015, sendo a maior inundação registrada em 2012 no Careiro da Várzea e em Beruri no ano de 2015.

As cotas fluviais dos municípios de Carauari, Sub-região do Juruá (Figura 2G), e Canutama, Sub-região do Purus (Figura 2J), obtiveram variações semelhantes. Outra similaridade está na quantidade de eventos extremos registrados nos dois municípios, total de onze. Sendo, cinco de inundações e seis de vazantes. As chuvas que ocorrem na região sudoeste da Amazônia Ocidental produzem aumento das vazões dos rios Purus e Juruá (Liberato, 2014). Dessa forma, essas chuvas contribuem diretamente com as cotas fluviais das sub-regiões homônimas que possuem características bem semelhantes, como a baixa variabilidade de suas cotas fluviais. Os municípios de Canutama (Purus) e Carauari (Juruá) obtiveram os menores valores de desvio padrão $35,6 \mathrm{~cm}$ e 53,5cm para máximas e $85,2 \mathrm{~cm}$ e 95,9cm para mínimas, respectivamente. A baixa variação das cotas fluviais nessas estações pode estar ligada às características geomorfológicas das áreas em questão.

No município de Santo Antônio do Içá, Sub-região do Alto Solimões, foram identificados treze eventos extremos, desses, sete foram de inundações e seis de vazantes. O Alto Solimões inicia-se na fronteira com a Colômbia, essa região é formada por dois vales afluentes à margem esquerda do rio principal: Içá e Japurá, bem como a margem direita pela complicada drenagem do rio Juruá (Ab'Sáber, 2010). Nessa região, o município de Santo Antônio do Içá possui uma concentração de eventos extremos de seca entre os anos de 1990 e 2000 e inundação entre os anos de 2009 e 2015.

Ao analisar a quantidade de eventos extremos hidrológicos fluviais por ano é possível verificar a maior frequência desses eventos (Figura 3). Os anos com maiores ocorrências de secas foram: 1998, 2005 e 2010. Já em relação a inundações os anos com maior número de registro foram: 2009, 2012, 2013, 2014 e 2015. Totalizando todos os eventos extremos se obtém um total de 113 eventos extremos hidrológicos ocorridos nas estações estudadas. Desses, 56 são de seca e 57 de inundação. 
Figura 3. Ocorrência de eventos extremos por ano nas estações analisadas do estado do Amazonas.

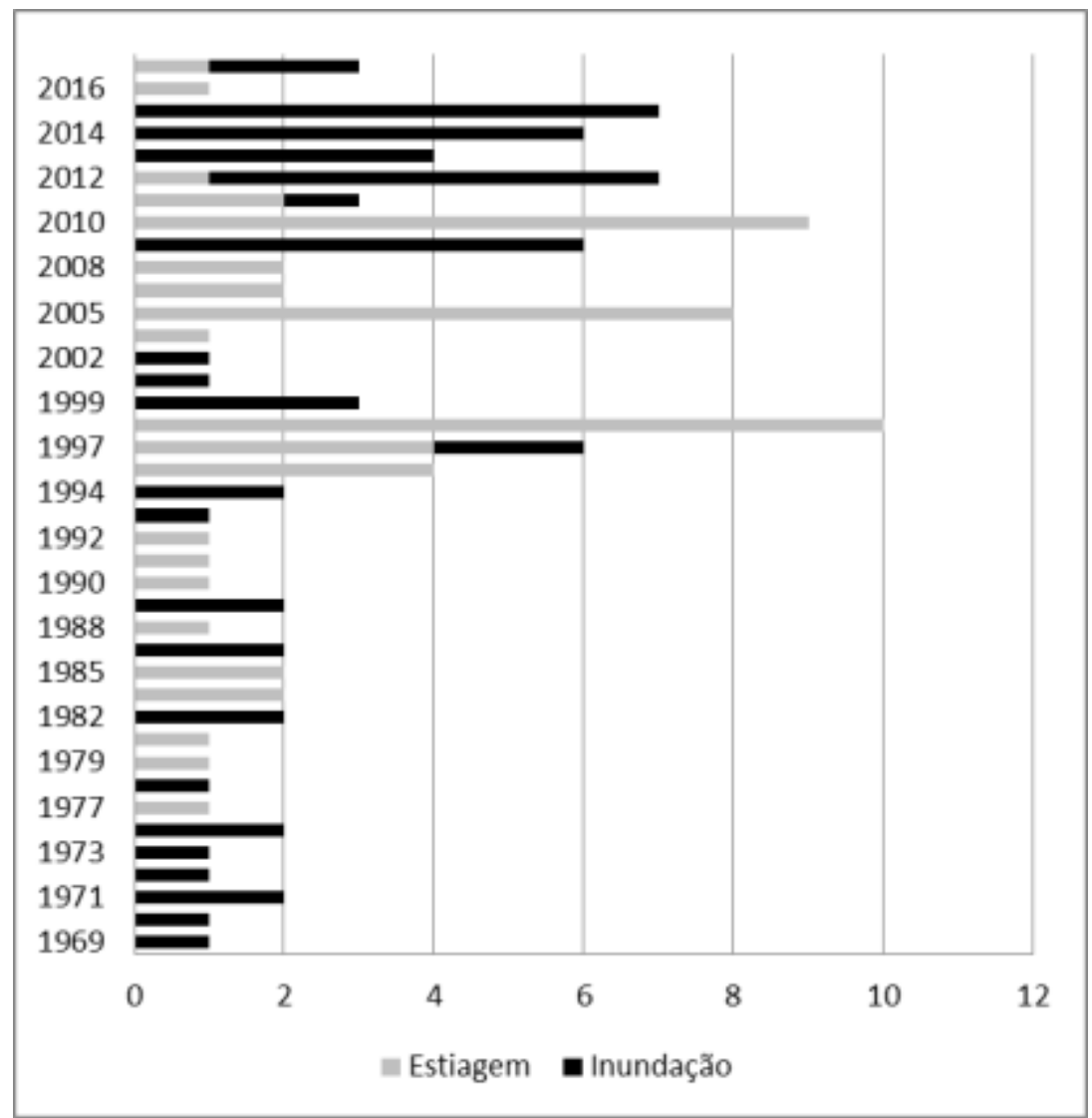

Fonte: Autores (2021).

As maiores frequências de eventos extremos fluviais de seca foram verificadas nos anos de 1997/1998, $2005 / 2006$ e 2009/2010. As maiores frequências de eventos extremos fluviais de seca foram verificadas nos anos de 1997/1998, 2005/2006 e 2009/2010. Diferente do ocorrido em 1998, as causas da seca de 2005 não foram ligadas pelo fenômeno El Niño, mas, sim ao aquecimento anômalo do Atlântico Norte Tropical (Marengo et al., 2008). De acordo com Liberato (2014):

Os anos de 1998, 2005 e 2010 foram classificados como anos muito secos. Os eventos de 1997/1998 e 2009/2010 apresentam características similares no Pacífico, com as condições de El Niño seguidas por uma rápida transição para condições de La Niña. Todos os três eventos foram marcados por TSM excessivamente quentes do Atlântico tropical norte. Particularmente em 2010, as temperaturas do mar atingiram os valores mais elevados na história na região do Atlântico tropical norte, que podem ter contribuído para o estabelecimento de uma circulação local meridional (célula de Hadley), com movimento vertical ascendente sobre o Atlântico norte e movimento vertical descendente (subsidência) sobre a Amazônia (Liberato, 2014, p.86).

Dessa forma, os eventos extremos de seca identificados no Amazonas têm relação com os padrões de circulação atmosférica potencializada por anomalias da temperatura da superfície do mar no Pacífico equatorial e Atlântico tropical norte (Liberato, 2014).

Os eventos de 2009 e 2012-2014 estiveram ligados com eventos de La Niña e/ou aumentos da temperatura da superfície do mar no Atlântico do Sul do Equador (Marengo \& Espinoza, 2016). Em 2014-2015, no sudoeste da Amazônia ocorreram severas inundações devido à ocorrência de precipitações acima do dobro de volume de anos normais (Espinoza et al., 2014). Na última década os eventos extremos de inundação ficaram mais frequentes no Amazonas, tornando mais vulnerável a vida da população ribeirinha no Amazonas (Barichivich et al., 2018).

As chuvas e a vazões dos rios sofrem também influências da Zona de Convergência Intertropical (ZCIT), da entrada 
de vapor advindo do Oceano Atlântico por ventos alísios e das trocas de calor e umidade entre vegetação e atmosfera na própria região (Marengo et. al., 2001; Tucci, 2002). Outro fator está ligado ao tamanho continental da bacia amazônica, levando-a a receber chuvas de maneira desigual, provocando a distribuição espacial heterogênea dos eventos extremos fluviais (Vale et al., 2011; Marengo et al., 2012).

\subsection{Os desastres ambientais no Amazonas}

De 2005 até 2017 a Secretaria Nacional de Proteção e Defesa Civil e o Subcomando de Ações de Defesa Civil do Amazonas reconheceram 427 desastres ambientais ocorridos no Amazonas (Figura 2). Desses, 358 são de Situação de Emergência e 69 de Estado de Calamidade Pública. A decretação do desastre indica as condições anormais geradas pela alteração grave ou intensa da situação de uma área (Brasil, 2012). A diferença entre os dois está justamente na capacidade resposta, pois a situação de calamidade indica o comprometimento parcial da capacidade de resposta e a calamidade pública indica o comprometimento substancial.

Figura 4. Ocorrência de desastres ambientais no Amazonas (2005-2017).
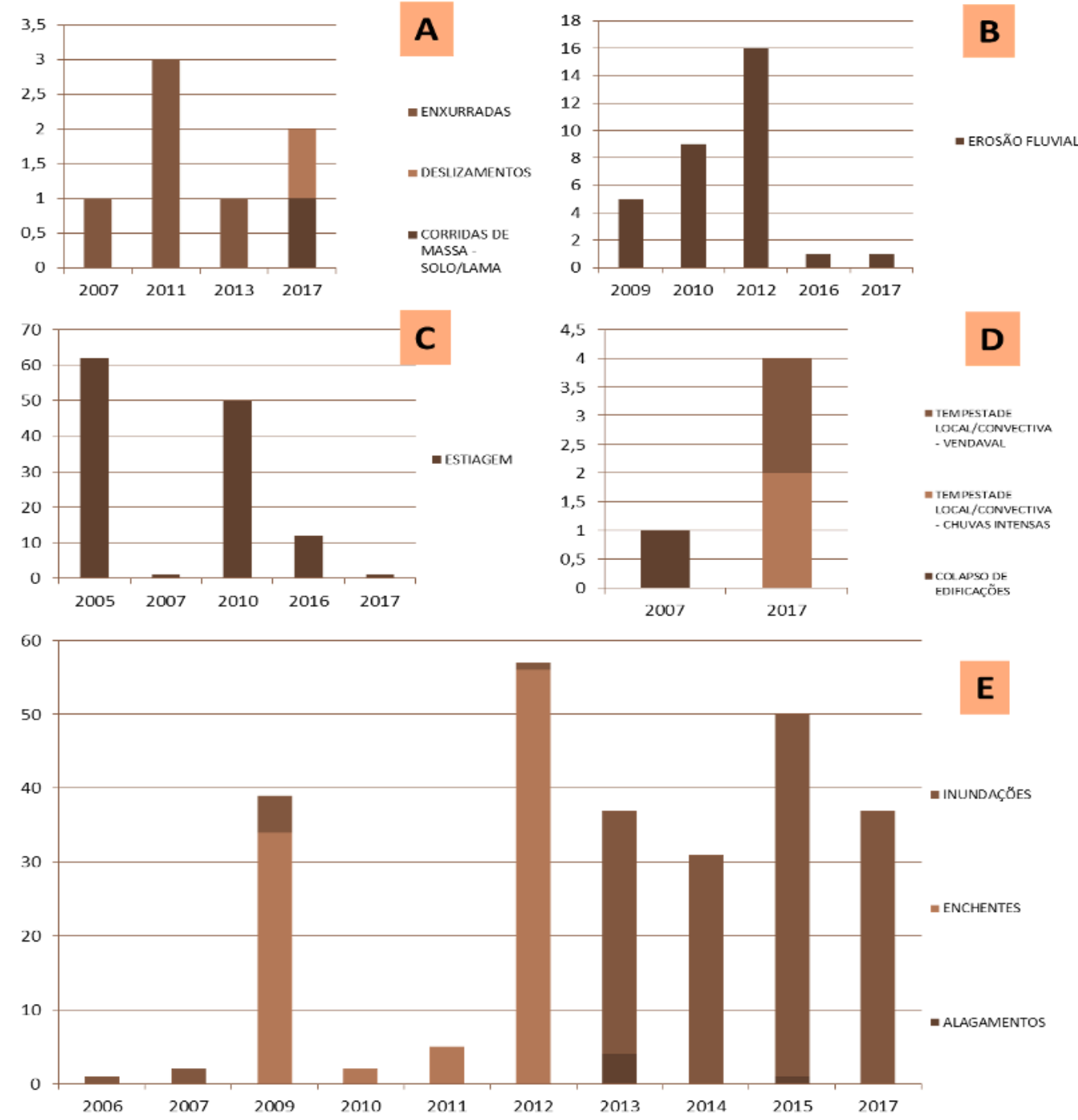

Fonte: Adaptado de informações obtidas da SENPDEC (2018). 
No período da análise temporal ocorreram cinco enxurradas, um deslizamento e uma corrida de massa (Figura 4A). A maior incidência de enxurradas foi em 2011 com a ocorrência de três enxurradas nos municípios de Carauari, Itacoatiara e Nova Olinda do Norte. Os desastres de erosão fluviais somam trinta e duas ocorrências no estado do Amazonas (Figura 4B).

As erosões fluviais geram impactos humanos importantes nas regiões de várzea, levando os comunitários a mudarem suas residências para locais mais distantes do rio ou mudarem para outras localidades. No ano de 2012 ocorreu o maior número de reconhecimento de desastres de erosão fluvial (Figura 4B). A sub-região com maior número de registros desse tipo de desastre é a da Região Metropolitana de Manaus com sete reconhecimentos, com destaque para o município de Manacapuru com três decretos reconhecidos.

Os vendavais, chuvas intensas e colapsos de edificações são eventos com poucos registros reconhecidos no estado do Amazonas (Figura 4D). O maior número de registros de vendavais e chuvas intensas foi realizado no ano de 2017, dois reconhecimentos cada. O baixo número desse tipo de desastres pode estar relacionado, além da pouca ocorrência, com a falta de informação dos governos municipais com os aspectos institucionais para a decretação desses desastres.

As vazantes (classificadas como estiagens) totalizam 126 reconhecimentos no período analisado (Figura 4C). A Região Metropolitana de Manaus obteve o maior número de registros desse tipo de desastre, vinte e três, seguida pela subregião do Triângulo Jutaí-Solimões-Juruá com quatorze reconhecimentos (Figura 5). O alto número de reconhecimentos realizados nos anos de 2005 e 2010 tem relação direta com os eventos extremos climáticos hidrológicos ocorridos na região no mesmo período (Marengo et al., 2008; Zeng et al., 2008; Liberato, 2014; Marengo \& Espinoza, 2016).

Figura 5. Desastres ambientais fluviais nas sub-regiões do Amazonas.

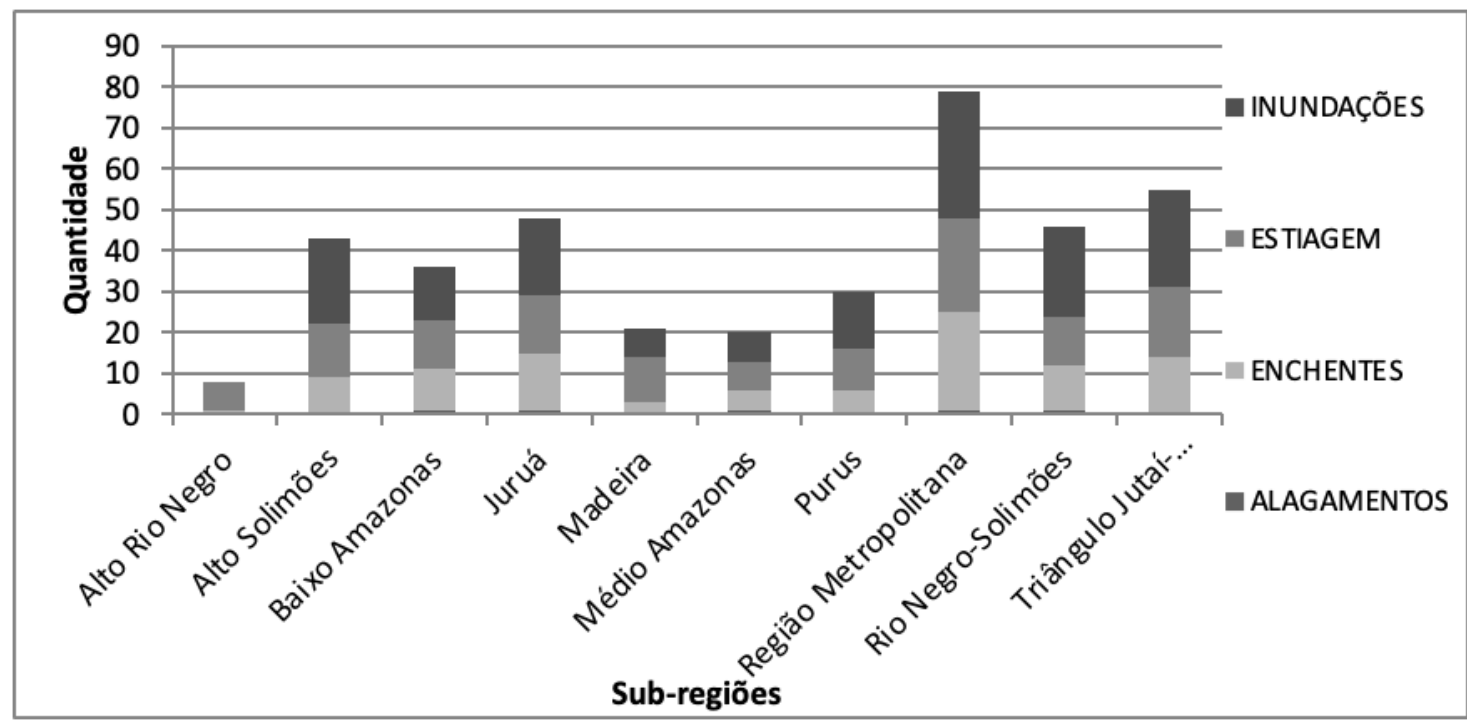

Fonte: Autores (2021).

Em relação aos registros de desastres ambientais, percebe-se uma maior quantidade de desastres nos anos que ocorreram eventos extremos fluviais (2005, 2010, 2012, 2013, 2014 e 2015). Dessa forma, no Amazonas 89,79\% dos desastres ocorridos desde 2005 têm relação com eventos fluviais (estiagem, inundações e erosões fluviais), totalizando trezentos e oitenta e seis (386).

A sub-região Metropolitana de Manaus detém o maior número de reconhecimentos de desastres ambientais de origem fluvial no Amazonas (Figura 5). Esse fato deve-se a localização dessa sub-região que recebe contribuição de várias calhas da bacia amazônica, bem como o número de municípios que existem na mesma. Os municípios de Careiro da Várzea e 
Manacapuru, ambos da Região Metropolitana de Manaus, estão com Ipixuna, Envira e Anamã entre os municípios com maior registro de desastres no estado, nove cada.

Com menor número de registros de desastres ambientais está o Alto Rio Negro que possui poucos municípios. Dentre os oito desastres reconhecidos nessa sub-região, sete foram de vazante e apenas um de inundação. A quantidade de desastres reconhecidos na sub-região do Alto Rio Negro (8) é inferior se comparada à quantidade de eventos extremos identificados apenas para o município de Barcelos (15). Nessa sub-região, nem todos os eventos que ocorrem tomam proporções de desastres ambientais fluviais. Um fator importante dessa sub-região é a baixa densidade populacional. Dessa forma, mesmo na ocorrência de um evento extremo fluvial o município acaba por não decretar situação de emergência ou estado de calamidade já que não houve importantes prejuízos sociais, econômicos e ambientais causados por esse evento. Isso se deve aos fatores culturais que influenciam essas populações a não ocuparem as áreas mais próximas as margens dos rios. Dessa forma, o fator ocupação acaba por ser um aspecto intrínseco para a ocorrência ou não de desastres ambientais.

Os desastres provocados pelas cheias no Amazonas são conhecidos pelo Sistema Integrado de Informação sobre Desastres (S2iD) de três formas diferentes: alagamento, enchente e inundação. Os alagamentos são definidos como um acúmulo momentâneo de águas gerado pela deficiência de sistema de drenagem; enchente está relacionada à elevação do canal do curso d'água, atingindo a cota máxima do canal, mas sem extravasá-lo; e, por fim, as inundações são definidas como o transbordamento de um curso d'água, atingindo a planície de inundação (Tominaga, 2009). Dessa forma, a denominação da COBRADE mais correta para esses desastres é a de inundação. Outros erros foram encontrados na codificação de quatro (4) inundações como erosões fluviais.

Dessa forma, os desastres não podem ser avaliados apenas pela existência de um perigo natural, mas, devem levar em consideração a vulnerabilidade socioambiental e a forma de resposta do poder público. Ao considerar essa interação, a forma mais correta de denominar esses desastres é classificá-los como ambientais.

A maioria dos decretos de desastres nas sub-regiões do Amazonas foi de inundação e suas diversas outras nomenclaturas, totalizando duzentos e setenta e dois (272) desastres ambientais fluviais. Entretanto, nas sub-regiões do Madeira e do Alto Rio Negro, a maior decretação foi de desastres de vazantes (classificados como estiagem) com onze e sete decretos, respectivamente.

\subsection{As cheias, secas e desastres no Amazonas}

De acordo com Ab'Sáber (1994), o regime pluviométrico rege os aspectos naturais e antrópicos na Amazônia. A dinâmica do regime hidrológico na Amazônia modifica o seu espaço, assim como sua paisagem, com regimes de secas e cheias, tal fato se denomina de sazonalidade (Abreu \& Oliveira, 2012). As áreas alagáveis na Amazônia Legal possuem quatro estações climáticas definidas pela sazonalidade dos rios: a enchente, a cheia, a vazante e a seca (Pereira, 2007).

A lentidão da enchente é um importante fator para a manutenção da biota nesses ecossistemas, além de ser imprescindível para existência de atividades produtivas (Sternberg, 1998; Pereira, 2007; Witkoski, 2010). As populações ribeirinhas da Amazônia possuem uma série de estratégias adaptadas ao ambiente físico dos trópicos e ao isolamento provocado pela precariedade das vias de transporte (Moran, 1974). Esse alto poder de adaptação se deve à plasticidade da resposta humana ao ambiente, em termos socioculturais e fisiológicos (Laraia, 1996; Moran, 1994).

As populações ribeirinhas têm como atividades econômicas e para consumo familiar a agricultura, o extrativismo, a atividade criatória e a pesca (Pereira, 2007; Sternberg, 1998; Witkoski, 2010). Essa polivalência é uma estratégia de adaptação às condições do meio, visto que atividades, como agricultura, não podem ser desenvolvidas durante todo o ano devido à dinâmica dos rios.

As mudanças sazonais das paisagens fluviais e da planície de inundação, como descritas anteriormente, são regulares 
e previsíveis, permitindo que os habitantes estabeleçam calendários definidos para as atividades sociais e produtivas, algo que tem se modificado nas últimas décadas. Dessa forma, as estratégias de adaptação humana nas regiões ribeirinhas da Amazônia são cada vez menos efetivas e eficientes frente à maior frequência dos eventos extremos climáticos fluviais.

Ao analisar a ocorrência de desastres ambientais fluviais e relacionar com os eventos climáticos extremos fluviais nos municípios estudados percebe-se importantes aspectos relacionados aos eventos climáticos extremos e a decretação de desastres na região (Figura 6).

Figura 6. Eventos extremos climáticos e ocorrência de desastres fluviais no Amazonas.

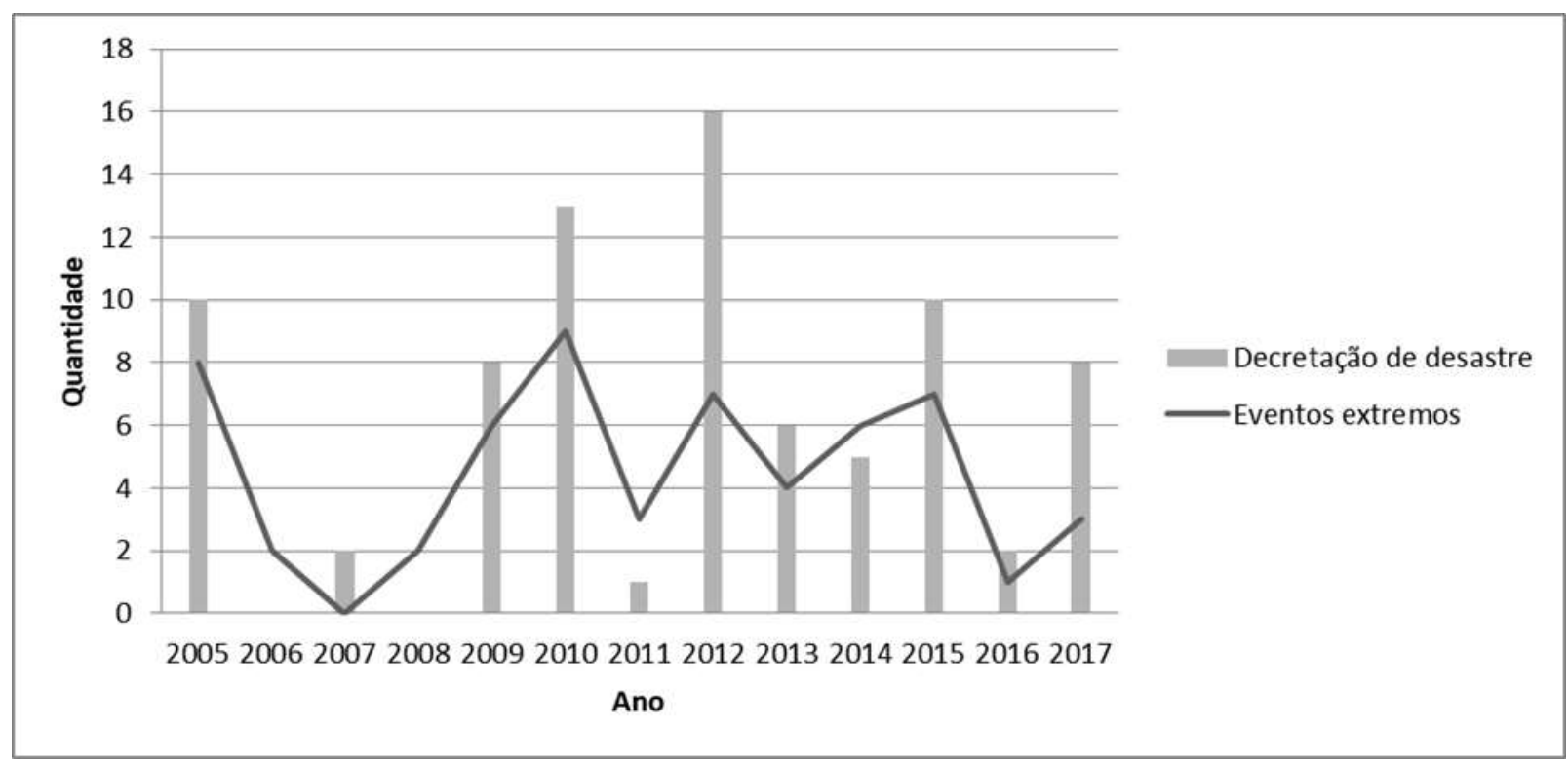

Fonte: Autores (2021).

Há uma forte ligação da ocorrência de eventos extremos climáticos fluviais com a decretação de desastres nas subregiões do estado do Amazonas. Esse fato fica evidente nos anos de 2005, 2009, 2010, 2012, 2013, 2014 e 2015 , cabe ressaltar que o início do reconhecimento desses eventos como desastres remonta o ano de 2005 (Rodrigues, 2013), onde os anos de maior número de decretos de desastres também são os de maior identificação de eventos extremos climáticos.

Entretanto, a decretação de desastres não ocorre apenas na existência de eventos extremos climáticos fluviais, visto que há decretação de desastres em anos que as cotas fluviométricas não superam os limiares de normalidade. Um claro exemplo está em municípios da Região Metropolitana de Manaus, como Iranduba que por possuir assentamentos humanos nas regiões de várzea e em baixas cotas possui problemas socioambientais frequentes, principalmente na subida dos rios Negro e Solimões (Guimarães, 2019).

A ocupação humana nas regiões inundáveis no Amazonas tem ligação com os aspectos culturais da formação social e cultural da população da região que sempre teve um forte vínculo e relação com os rios da região (Tocantins, 2000). Entretanto, outro fator que tem crescido muito em regiões mais urbanizadas no Amazonas está na ocupação irregular de regiões próximas aos corpos hídricos nas cidades por serem os únicos locais disponíveis para pessoas de baixa renda, sem garantia de políticas públicas básicas como saneamento, tornando-se locais de alta vulnerabilidade socioambiental (Alves, 2006).

Para além dos impactos dos eventos extremos climáticos e eventos próximos aos limiares de normalidade, outro fator que gera impactos na decretação de desastres naturais está na capacidade dos municípios e das coordenadorias municipais de 
proteção e defesa civil realizarem o preenchimento do formulário de informações de desastres e o próprio processo de decretação desses desastres.

A ocorrência de eventos extremos climáticos fluviais, bem como eventos próximos aos limiares extremos de cheia e seca, está cada vez mais frequentes devido a mudança climática global. A própria comunidade científica já reconhece que com o advento da mudança climática global, o risco e ocorrência de eventos extremos tende a se intensificar (IPCC, 2014; Barichivich et al., 2018).

\section{Considerações Finais}

No estado do Amazonas a água sempre foi reguladora do cotidiano das populações ribeirinhas e detém a centralidade da relação com o ambiente. Ao realizar a análise de uma estação por sub-região foi possível verificar as alterações no regime hidrológico fluvial que estão ocorrendo no Amazonas, através da maior frequência de eventos extremos hidrológicos fluviais. Entretanto, devido o tamanho do Estado é necessário verificar e analisar mais estações distribuídas nas diferentes sub-regiões. A interação desses perigos naturais com a vulnerabilidade das populações no Amazonas vem gerando um aumento dos desastres ambientais. Nesse sentido, há um aumento de registros de desastres em anos com ocorrência de eventos extremos hidrológicos fluviais, de inundação e seca. Entretanto, os eventos hidroclimáticos não precisam atingir o limiar de extremo para ser considerado um desastre ambiental fluvial no Amazonas, visto que algumas populações já sofrem danos materiais e econômicos na faixa de normalidade dos eventos, devido sua vulnerabilidade socioambiental e residência em cotas muito baixas.

Existem discrepâncias na nomenclatura dos desastres ambientais a partir da Codificação Brasileira de Desastres (COBRADE) no Amazonas devido à falta de uma codificação específica para inundações graduais existentes na Amazônia, bem como a baixa orientação dos colaboradores nos municípios no preenchimento do Formulário de Identificação de Desastres (FIDE).

De forma geral, houve uma maior frequência de eventos extremos fluviais em todo o estado do Amazonas nos últimos anos. Com destaque para os anos de 1998, 2005 e 2010 para seca e 2009, 2012, 2013, 2014 e 2015 para inundação. A ocorrência desses eventos extremos e de eventos próximos a esses limiares teve como resultado uma maior ocorrência de desastres ambientais fluviais de inundação e seca no Estado. No período analisado, a Região Metropolitana de Manaus obteve o maior número de decretações de desastres.

A complexidade do sistema hidrológico no Amazonas não pode ser atribuída a causas isoladas, mas deve considerar a existência de diversos fatores para se explicar a ocorrência desses eventos extremos. Dessa forma, todas as sub-regiões do estado do Amazonas já estão sofrendo as implicações socioambientais provenientes da mudança climática na sazonalidade dos rios que geram uma maior frequência dos eventos extremos hidrológicos e dos consequentes desastres ambientais fluviais

\section{Referências}

Ab' Saber, A. N. (1994). Amazônia Brasileira: Um Macro Domínio. Amazônia, Flora e Fauna, Rio de Janeiro, Alumbramento.

Ab' Saber, A. N. (2010). Zoneamento fisiográfico e ecológico do espaço total da Amazônia Brasileira. Est. ava., São Paulo, v. 24 , n. 68, p. 15-24.

Abreu, R. P. \& Oliveira, H. S. (2012). A influência das mudanças sazonais nos aspectos naturais e sociais no careiro da várzea - Am. Eixo temático: clima, ambiente e atividades rurais. Revista geonorte, Edição Especial 2, V.2, N.5, p.1399 - 1408.

Alves, H. P. da F. (2006). Vulnerabilidade socioambiental na metrópole paulistana. Revista brasileira de estudos populacionais, São Paulo, v. 23 , n. 1.

Barichivich, J.; et al. (2018). Recent intensification of Amazon flooding extremes driven by strengthened Walker circulation. Science Advances. Vol. 4, no. 9. DOI: $10.1126 /$ sciadv.aat8785

Basher, R. (2008). Disaster impacts: implications and policy responses. Social Research: an International Quarterly. v. 75, n. 3, p. 937-954. 
Brasil. (2012). Instrução Normativa Ministério da Integração Nacional, N. 1. Estabelece procedimentos e critérios para a decretação de situação de emergência ou estado de calamidade pública pelos Municípios, Estados e pelo Distrito Federal, e para o reconhecimento federal das situações de anormalidade decretadas pelos entes federativos e dá outras providências.

Brasil. (2012). Ministério da Integração Nacional. Secretaria Nacional de Defesa Civil. Centro Nacional de Gerenciamento de Riscos e Desastres. Anuário brasileiro de desastres naturais. Centro Nacional de Gerenciamento de Riscos e Desastres.

Carvalho, D. W. (2013). As mudanças climáticas e a formação do direito dos desastres. Novos Estudos Jurídicos, v. 18, n. 3, p. $397-415$.

Da Silva Dias, M. A.F. (2014.). Eventos climáticos extremos. Revista USP, n. 103, p. 33-40.

Espinoza, J. C.; et al. (2014). The extreme 2014 flood in South-Western Amazon basin: the role of tropical-subtropical South Atlantic SST gradient. Environ. Res. Lett. 9: 124007, doi: 10.1088/1748-9326/9/12/124007.

Filizola, N. P.; et al. (2006). Cheias e secas na Amazônia: Breve abordagem de um contraste na maior bacia hidrográfica do globo. T\&C Amazônia, v. 9.

Fraxe, T. J. P; Pereira, H.S. \& Witkoski, A. C. (2007). Comunidades ribeirinhas amazônicas - modos de vida e uso dos recursos naturais. EDUA.

Fraxe, T.J.P. (2000). Homens anfíbios: etnografia de um campesinato das águas. Annablume; Fortaleza: Secretaria de Desporto do Governo do Estado do Ceará.

Freitas, C. M.; et al. (2014). Desastres naturais e saúde: uma análise da situação do Brasil. DOI: 10.1590/1413-81232014199.00732014.

IPCC. Intergovernmental Panel on Climate Change Climate Change (2007). The physical science basis (summary for policymakers).

Jardim-Lima, D.; et al. (2005). A dinâmica do Pulso de inundação: aplicações de sensoriamento remoto na avaliação da área de águas abertas e morfologia dos lagos de várzea da Reserva de Desenvolvimento Sustentável Mamirauá-Ammazônia Central. XII Simpósio Brasileiro de Sensoriamento Remoto, Goiânia/INPE, p. 3069-3076,

Laraia, R. de B. (1996). Cultura - Um Conceito Antropológico. 11. a edição. Jorge Zahar Editor

Liberato, A. M. (2014). Estudo de Eventos Climáticos Extremos na Amazônia Ocidental e seus Impactos na Hidrovia Rio Madeira. Tese (Doutorado em Meteorologia) - Universidade Federal de Campina grande. Campina Grande.

Marengo J. A.; et al. (2012). Extreme climatic events in the Amazon basin. Climatological and hydrological context of recent floods. Theor Appl Climatol. 107:73-85.

Marengo J. A.; et al. (2008). Hydro-Climatic and Ecological Behaviour of the Drought of Amazonia in 2005. Philosophical Transactions of The Royal Society, 363:1773-1778

Marengo, J. A. \& Espinoza, J. C. (2016). Extreme seasonal droughts and floods in Amazonia: causes, trends and impacts. International Journal of Climatology, v. 36, n. 3, p. 1033-1050.

Marengo, J. A.; et al. (2001). Onset and end of the rainy season in the Brazilian Amazon Basin", J. Clim., 14, 833-852.

Moran, E. F. (1974). A Ecologia Humana das Populações da Amazônia.

Moran, E. F. (1994). Adaptabilidade Humana: Uma Introdução à Antropologia Ecológica. Editora da Universidade de São Paulo.

Muniz, L. \& Filizola, N. (2014). A influência da geomorfologia na variabilidade fluviométrica: o caso do Rio Madeira - Brasil. Revista Geonorte, Edição Especial 4, V.10, N.4, p.6-10, (ISSN 2237-1419).

Naghettini, M. \& Pinto, E. J. A. (2007). Hidrologia estatística. CPRM.

Nascimento, D. G. (2016). Entre a terra e a água: modo de vida camponês no médio Rio Amazonas, Parintins-AM. Dissertação de Mestrado (Mestrado em Geografia) - Universidade Federal do Amazonas.

Oliveira, E.; et al. (1994). Hidrologia da bacia do Rio Amazonas. Água em Revista. CPRM.

Pereira, H. P. (2007). A dinâmica socioambiental das Várzeas do rio Solimões-Amazonas.

Rodrigues, A. S. B. (2013). Jornalismo e meio ambiente na Amazônia: a cobertura de eventos ambientais extremos pela imprensa escrita de Manaus. 203 f. Tese de Doutorado (Doutorado em Sociedade e Cultura) - Universidade Federal do Amazonas.

Saito, S. M.; Soriano, E. \& Londe, L. de. R. (2015). Desastres naturais. São Paulo: Oficina de textos.

Sausen, T. M. \& Lacruz, M. S. P. (2015). Sensoriamento Remoto para desastres. São Paulo: Oficina de textos.

Sternberg, H. O. R. (1998). A Água e o Homem na Várzea do Careiro. Museu Paraense Emílio Goeldi. Belém.

Tocantins, L. (2000). O rio comanda a vida: uma interpretação da Amazônia. $9^{a}$ edição. Editora Valer/Edições Governo do Estado.

Tominaga, L. K. (2009). Desastres Naturais: Por que ocorrem? São Paulo: Instituto Geológico.

Tominaga, L. K.; Santoro, J. \& Do Amaral, R. (2009). Desastres naturais - conhecer para prevenir. Instituto Geológico.

Tucci, C. E. M. (2002). Impactos da variabilidade climática e uso do solo sobre os recursos hídricos. Agência Nacional de Águas. 
Research, Society and Development, v. 10, n. 9, e25510917882, 2021

(CC BY 4.0) | ISSN 2525-3409 | DOI: http://dx.doi.org/10.33448/rsd-v10i9.17882

UNISDR. International Strategy for Disaster Risk Reduction. (2009). UNISDR terminology on disaster risk reduction.

Vale, R. do.; et al. (2011). A cheia de 2009 na Amazônia Brasileira. Revista Brasileira de Geociências, Vol. 41 (4): $577-586$.

Valêncio, N. F. L. da S. (2014). Disasters: technicism and social suffering. Ciência \& Saúde Coletiva, v. 19, n. 9, p. 3631-3644.

Vieira, G. S.; Peixoto, A. S. P. \& Kaiser, I. M. (2016). Análise da Estrutura dos Bancos de Dados de Desastres no Estado de São Paulo.

Witkoski, A. C. (2010). Terras, florestas e águas de trabalho: os camponeses amazônicos e as formas de uso de seus recurso naturais. Annanblume.

Zeng, N. (2008). Causes and impacts of the 2005 Amazon drought. Environ. Res. Lett. 3: 014002, doi: 10.1088/1748-9326/3/1/014002. 